\title{
Participação Popular no Contexto das Iniciativas de Governo Aberto: revisão sistemática da literatura
}

\section{Popular Participation in the Context of Open Government Initiatives: a systematic review of the literature}

\author{
Cristiane S. Sanchez \\ Universidade Federal do Paraná (UFPR) - Programa de Pós-Graduação em Ciência, Gestão e \\ Tecnologia da Informação \\ E-mail: cristiane.sinimbu@ufpr.br \\ Patrícia Z. Marchiori \\ Universidade Federal do Paraná (UFPR) - Programa de Pós-Graduação em Ciência, Gestão e \\ Tecnologia da Informação \\ E-mail: pzeni@ufpr.br
}

Resumo: As iniciativas de Governo Aberto (GA) preconizam a transparência, a colaboração e a inovação, o controle social e a participação como formas de combate à corrupção e aprimoramento da gestão democrática. Este estudo buscou explorar os elementos que caracterizam a participação popular no contexto das iniciativas de Governo Aberto, derivando-se da aplicação de alguns protocolos de revisão sistemática da literatura, estruturado com base em estratégia de busca nas bases de dados: Business Source Complete, Science Direct e Web of Science - Coleção Principal. Aplicaram-se critérios de inclusão/exclusão de documentos, resultando em 64 documentos para análise. Os resultados indicam que a participação, no contexto de GA, compõe-se de alguns elementos, a saber: espaços, ferramentas, tecnologias de informação e comunicação. A participação se dá, por vezes, de forma institucionalizada, sustentada por entidades coletivas que representam a sociedade civil organizada. Quanto à participação dos cidadãos na elaboração destas políticas, as iniciativas de GA se encontram em estágio de implementação e reformulação de políticas que garantam tanto o acesso aos dados abertos governamentais, assim como os espaços de representação da sociedade. Aponta-se, ainda, que existem condicionantes para a participação - barreiras e desafios - que estão relacionadas à assimetria de informação, falta de experiência no uso das ferramentas, entre outros. Destaca-se que a sistematização destes elementos pode contribuir para a melhoria de políticas de inclusão da sociedade na elaboração e acompanhamento de políticas públicas de interesse dos cidadãos. Considera-se que a apresentação e discussão destes elementos possa contribuir, futuramente, para a criação um quadro conceitual na temática.

Palavras-Chave: Participação cidadã; Governo Aberto; formas de participação; Revisão Sistemática

Abstract: The Open Government initiatives advocate transparency, collaboration and innovation, accountability and participation as ways of combating corruption and improve democratic 
management. This study explores the elements that characterize popular participation in the context of Open Government initiatives, deriving from the application of some protocols of systematic review, structured based on search strategy in databases: Business Source Complete, Science Direct and Web of Science. By apply document inclusion/exclusion criteria 64 documents were obtained, wich indicate that participation, in this context, is composed by some elements, namely: spaces, tools, information and communication technologies. Participation sometimes occurs in an institutionalized way, supported by collective entities that represent civil society. Regarding the effective participation of citizens in the elaboration of these policies, Open Government initiatives are in the stage of implementation and reformulation of policies that guarantee both the access to open data and government information, as well as the spaces of representation of society. Another important element is motivation, which is related to the possibility of accountability and to greater governmental transparency. The results also point out constraints to effective participation, namely: barriers and challenges, which are related to information asymmetry, lack of experience in the use of tools, among others. It should be noted that the systematization of these elements may contribute to improve policies for the inclusion of society in the elaboration and monitoring of public policies of interest to citizens. The presentation and discussion of these elements may also contribute, in the future, to the creation of a conceptual framework on the subject.

Keywords: Citizen participation; Open Government; forms of participation; Systematic Review.

\section{Introdução}

Ao longo das últimas décadas vem-se construindo um movimento voltado à abertura dos governos de maneira a dar suporte ao combate à corrupção, promover a transparência e a participação popular na gestão pública (Yu \& Robinson, 2012). Até o ano de 2016, setenta países se juntaram à Open Government Partnership ${ }^{1}$ com o intuito de promover uma parceria entre os signatários para o comprometimento com políticas de transparência, empoderamento do cidadão,

\footnotetext{
${ }^{1}$ A Open Government Partnership - OGP (Parceria para Governo Aberto, em português) é uma iniciativa internacional que pretende difundir e incentivar globalmente práticas governamentais relacionadas à transparência dos governos, ao acesso à informação pública e à participação social. A OGP foi lançada em 20 de setembro de 2011, quando os oito países fundadores da Parceria (África do Sul, Brasil, Estados Unidos, Filipinas, Indonésia, México, Noruega e Reino Unido) assinaram a Declaração de Governo Aberto e apresentaram seus Planos de Ação. Atualmente, 75 países integram a Parceria. (Com informações disponíveis em: http://www.governoaberto.cgu.gov.br/a-ogp/o-que-e-ainiciativa. Acesso em: 12 jul. 2017).
}

Revista Brasileira de Políticas Públicas e Internacionais, v.2, n.2, Dezembro/2017, pp. 103-118. 
combate à corrupção e desenvolvimento de novas tecnologias que fortaleçam a governança ${ }^{2}$ (Open Government Declaration, 2011).

Estes compromissos, firmados por meio de documentos ${ }^{3}$, têm como pilares a transparência, o controle e prestação de contas, a colaboração e inovação; e são centrados no envolvimento de cidadãos no processo de tomada de decisão. Neste sentido, os princípios fundamentais deste Movimento são: a) a disponibilização de informações governamentais e a implementação de padrões profissionais para ocupantes da alta gestão; b) o aumento do acesso a novas tecnologias para abertura e controle das contas públicas; e principalmente, c) a promoção da participação popular (Open Government Declaration, 2011).

A participação popular, no que tange à elaboração de políticas embasadas em dados e informações públicas governamentais ou não (entre outras ações e possibilidades) se destaca como um dos fatores de transformação social. Os relatórios emitidos pelas organizações e instituições que acompanham as iniciativas de Governo Aberto (GA), as políticas de dados abertos e o acesso à informação, têm ilustrado este cenário de participação popular (Foti, [2014]).

O conceito de participação popular envolve múltiplas interpretações, podendo abarcar desde o envolvimento em alguma atividade, em entidades e coletivos, até a organização de uma agenda de trabalho para a tomada de decisão em organizações, as quais podem impactar os cidadãos diretamente, consolidando-se como fator determinante para que os Governos se tornem mais transparentes, responsáveis e sensíveis às demandas de seus próprios cidadãos (Rowe \& Frewer, 2005).

\footnotetext{
${ }^{2} \mathrm{O}$ conceito de governança evoluiu para identificar e explicar novos modos de resolução de problemas e tomada de decisão que preencham lacunas criadas pelo fracasso das formas tradicionais. Apresenta-se como uma maneira mais flexível e democrática de lidar com problemas públicos. FISHER, F. Participatory governance as deliberative empowerment: the cultural politics of discursive space. American Review of Public Administration, v. 36, n. 1, 2006. Disponível em: <http://journals.sagepub.com/doi/abs/10.1177/0275074005282582>. Acesso em: 01 mar. 2017.

${ }^{3}$ Open Government Declaration; Carta Latino-Americana de Governo Aberto; Tratado de Aarhus, Open Charter, entre outros.
}

Revista Brasileira de Políticas Públicas e Internacionais, v.2, n.2, Dezembro/2017, pp. 103-118. 
Em outra perspectiva, Gaventa \& Valderrama, $1999^{4}$ apresentam a participação sob a ótica social - entendida como a relação entre uma organização pública e a sociedade; e política - que se manifesta de duas formas: a) indireta, por meio de processos eleitorais na escolha de representantes; e, b) direta, via a interação com as organizações públicas de modo a contribuir para elaboração de políticas. A discussão sobre espaços de fala garantidos à população, perpassando espaços de escuta por parte dos gestores públicos, é reforçada por (Heredia, Bezerra, \& Palmeira, 2012), que ampliam o conceito de participação popular, ressaltando que esta se dá nos espaços de fala institucionalizados, por meio das distintas formas de organização, ou ainda, por meio do acesso efetivo a informações governamentais e às discussões de prioridades dos governos pelos cidadãos.

Percebe-se, assim, que os elementos que caracterizam a participação popular se apresentam de forma multifacetada e, portanto, uma sistematização destes elementos pode auxiliar os interessados em promover a participação popular no contexto das iniciativas de Governo Aberto, reforçando as premissas que o envolvem.

Neste sentido, buscou-se identificar os elementos que, em conjunto, caracterizam a participação popular no contexto das iniciativas de Governo Aberto, com base em estudos teóricos e empíricos divulgados na literatura sobre o tema.

\section{Procedimentos Metodológicos}

A Revisão Sistemática (RS) tem suas origens na área de Ciências da Saúde, elaborada principalmente pela rede Cochrane ${ }^{5}$ de pesquisadores, profissionais, pacientes e interessados na área de saúde, e é utilizada para identificar, avaliar e sintetizar todas as evidências empíricas que atendam aos critérios de elegibilidade pré-especificados por investigadores para responder a uma determinada pergunta de pesquisa (The Cochrane Database of Systematic Reviews, 2017).

\footnotetext{
${ }^{4}$ Citado por Silva (2015).

${ }^{5}$ http://www.cochrane.org/
}

Revista Brasileira de Políticas Públicas e Internacionais, v.2, n.2, Dezembro/2017, pp. 103-118. 
SANCHEZ, Cristiane S. e MARCHIORI, Patrícia Z. A. Participação Popular no Contexto das iniciativas de Governo Aberto: revisão sistemática da literatura

Em 2004, Kitchenham, baseando-se nesta metodologia, adaptou-a para a área de Engenharia de Software e, em 2007, Dybå, Dingsøyr e Hanssen incluíram nesta adaptação passos para avaliação e análise dos dados resultantes da aplicação dos termos de busca nas bases de dados (Attard, Orlandi, Scerri, \& Auer, 2015).

Em 2015, (Attard et al., 2015) ajustaram tais adaptações para a área de Ciências Humanas, utilizando-a no levantamento das iniciativas de dados abertos governamentais. Para os autores, a RS consiste em um estudo aprofundado, replicável por qualquer pesquisador, de determinado tema norteado por uma questão problema para a pesquisa, à qual se agregam questões secundárias. Uma revisão sistemática aplica critérios de inclusão e exclusão de material para análise, assim como a seleção de bases de dados a serem consultadas, nas quais as estratégias de buscas são definidas de forma a cobrir o máximo da literatura disponível e, ainda, evitar vieses por parte do pesquisador. Esta adaptação foi a utilizada como a abordagem metodológica para esta investigação (Attard et al., 2015).

Considerando-se o objetivo do estudo, a etapa de formulação da questão de pesquisa - que inicia o ciclo da RS - resultou na seguinte pergunta:

\section{$Q^{1}$ - Quais os elementos que caracterizam a participação popular no contexto das iniciativas de Governo Aberto?}

As questões secundárias, que neste particular possibilitam o direcionamento para análise dos documentos, foram: 1) quais são as condicionantes (desafios/barreiras) para participação popular?; e, 2) quais são os impactos da participação popular nas iniciativas de governo aberto?

Para a etapa de definição da estratégia de busca, considerou-se que as expressões “participação popular” e “governo aberto” seriam empregadas na composição das funções booleanas a serem inseridas nas caixas de busca das bases de dados selecionadas. No idioma Inglês a expressão de busca foi configurada como ((("civi* participation" OR "popular participation" OR "citiz* participation")) AND (("open government"))), com sua tradução para Português e Espanhol.

Revista Brasileira de Políticas Públicas e Internacionais, v.2, n.2, Dezembro/2017, pp. 103-118. 
SANCHEZ, Cristiane S. e MARCHIORI, Patrícia Z. A. Participação Popular no Contexto das iniciativas de Governo Aberto: revisão sistemática da literatura

Para seleção das bases de dados, levou-se em consideração a cobertura nas áreas de Ciências Sociais (Sociologia, Ciências Políticas, Humanidades), Administração Pública, assim como bases multidisciplinares, disponíveis no Portal do Sistema de Bibliotecas da Universidade Federal do Paraná (UFPR) ${ }^{6}$. As bases selecionadas foram: Business Source Complete (EBSCOHos), Science Direct (Elsevier) e Web of Science - Coleção Principal (Thomson Reuters Scientific).

Os resultados derivados das buscas (202 documentos) foram incluídos no Gerenciador de Referências EndNote ${ }^{\mathrm{TM}}$ basic (Thomson Reuters). Retiraram-se as duplicatas identificadas tanto pelo gerenciador como de forma manual, sendo que o corpus final do estudo resultou em 186 documentos, conforme ilustra a Tabela 1.

Tabela 1: Resultado das buscas nas Bases de Dados na temática "participação social” e "governo aberto"

\begin{tabular}{|c|c|c|c|c|}
\hline \multirow[t]{2}{*}{ Bases de Dados } & \multicolumn{3}{|c|}{ Resultados } & \multirow[t]{2}{*}{ Total } \\
\hline & $\begin{array}{l}\text { ((("civi* } \\
\text { participation" } \\
\text { OR "popular } \\
\text { participation" } \\
\text { OR "citiz* } \\
\text { participation")) } \\
\text { AND (("open } \\
\text { government"))) }\end{array}$ & $\begin{array}{l}\text { ((("participação } \\
\text { civi*" OR } \\
\text { "participação } \\
\text { popular" OR } \\
\text { "participação } \\
\text { cidad*")) AND } \\
((\text { "governo } \\
\text { aberto"))) }\end{array}$ & $\begin{array}{l}\text { ((("participación } \\
\text { cívica" } \\
\text { "participación } \\
\text { popular" } \\
\text { "participacion } \\
\text { ciudadana")) AND } \\
((\text { ("gobierno } \\
\text { abierto"))) }\end{array}$ & \\
\hline $\begin{array}{l}\text { Web of Science - } \\
\text { Coleção Principal } \\
\text { (Thomson Reuters } \\
\text { Scientific) }\end{array}$ & 20 & 0 & 0 & 20 \\
\hline $\begin{array}{l}\text { Business Source } \\
\text { Complete (EBSCOHost) }\end{array}$ & 36 & 2 & 5 & 43 \\
\hline $\begin{array}{l}\text { Science Direct } \\
\text { (Elsevier) }\end{array}$ & 133 & 1 & 5 & 139 \\
\hline Total & 189 & 3 & 10 & 202 \\
\hline $\begin{array}{l}\text { Trabalhos duplicados - v } \\
\text { Trabalhos duplicados - v } \\
\text { Corpus final do estudo }\end{array}$ & $\begin{array}{l}\text { rificação do EndN } \\
\text { rificação manual }\end{array}$ & $\mathrm{e}^{\mathrm{TM}}$ & & $\begin{array}{r}06 \\
10 \\
186\end{array}$ \\
\hline
\end{tabular}

Fonte: Elaborado pelas autoras (2016).

${ }^{6}$ http://www.portal.ufpr.br/bases_restritas.html

Revista Brasileira de Políticas Públicas e Internacionais, v.2, n.2, Dezembro/2017, pp. 103-118. 
A etapa de inclusão/exclusão de estudos foi dividida em duas fases. A primeira considerou os procedimentos de análise de conteúdo elencados por (Bardin, 2016) pré-selecionando-se os documentos a partir dos títulos e resumos dos 186 documentos recuperados. Quando surgiram dúvidas sobre o teor de alguns materiais fez-se uma leitura flutuante ${ }^{7}$ dos textos completos, respeitando-se a orientação da autora voltada à exaustividade, representatividade, pertinência e homogeneidade do corpus de estudo. Importa destacar que tais critérios implicaram na não adoção de um recorte temporal, e tampouco restrições iniciais quanto ao tipo de material.

A segunda fase consistiu na definição de critérios de inclusão/exclusão de documentos, que respeitou a decisão de que o corpus de análise seria composto apenas por documentos que: a) explicitamente indicassem a presença de discussão acerca de participação popular e/ou governo aberto; ou, b) que tratassem de dados abertos governamentais, movimentos sociais, representações sociais, tecnologias de informação e comunicação em contexto governamental e/ou de participação. Os documentos que não apresentaram tais conteúdos foram excluídos do corpus, assim como os editoriais; outros itens identificados como conferências/palestras; ou, ainda, documentos aos quais não se teve acesso ao texto completo, Dois documentos, um em italiano e outro em japonês, foram descartados de forma a garantir a consistência com as questões de busca. Embora não se tenha definido qualquer restrição por tipo de material e arco temporal, excluíram-se um livro e quatro capítulos de livros, pois não se teve acesso aos documentos em texto completo.

Após aplicação dos critérios de inclusão e exclusão, dos 186 documentos recuperados, sessenta e quatro (64) foram separados para análise, dos quais 59 são artigos de periódicos (quatro no prelo à época da coleta) e cinco comunicações em eventos. Destes, três no idioma espanhol e 61 no idioma inglês. Quanto ao tipo de estudo, o corpus se divide em 26 estudos empíricos, 22 estudos de caso e dezesseis revisões de literatura.

\footnotetext{
${ }^{7}$ A leitura flutuante consiste em estabelecer contato com os documentos a analisar, suscitando as impressões e orientações dos documentos (Bardin, 2016).
}

Revista Brasileira de Políticas Públicas e Internacionais, v.2, n.2, Dezembro/2017, pp. 103-118. 
SANCHEZ, Cristiane S. e MARCHIORI, Patrícia Z. A. Participação Popular no Contexto das iniciativas de Governo Aberto: revisão sistemática da literatura

\section{Discussão dos Resultados}

A leitura flutuante fez emergir as unidades de sentido e de contextualização, ilustradas na Figura 1, entendidas como elementos sumários que oportunizam uma prévia categorização, assim como o entendimento acerca da temática tratada nos documentos (Bardin, 2016).

Figura 1. Unidades de registro e contexto emergentes da leitura dos resumos dos documentos.

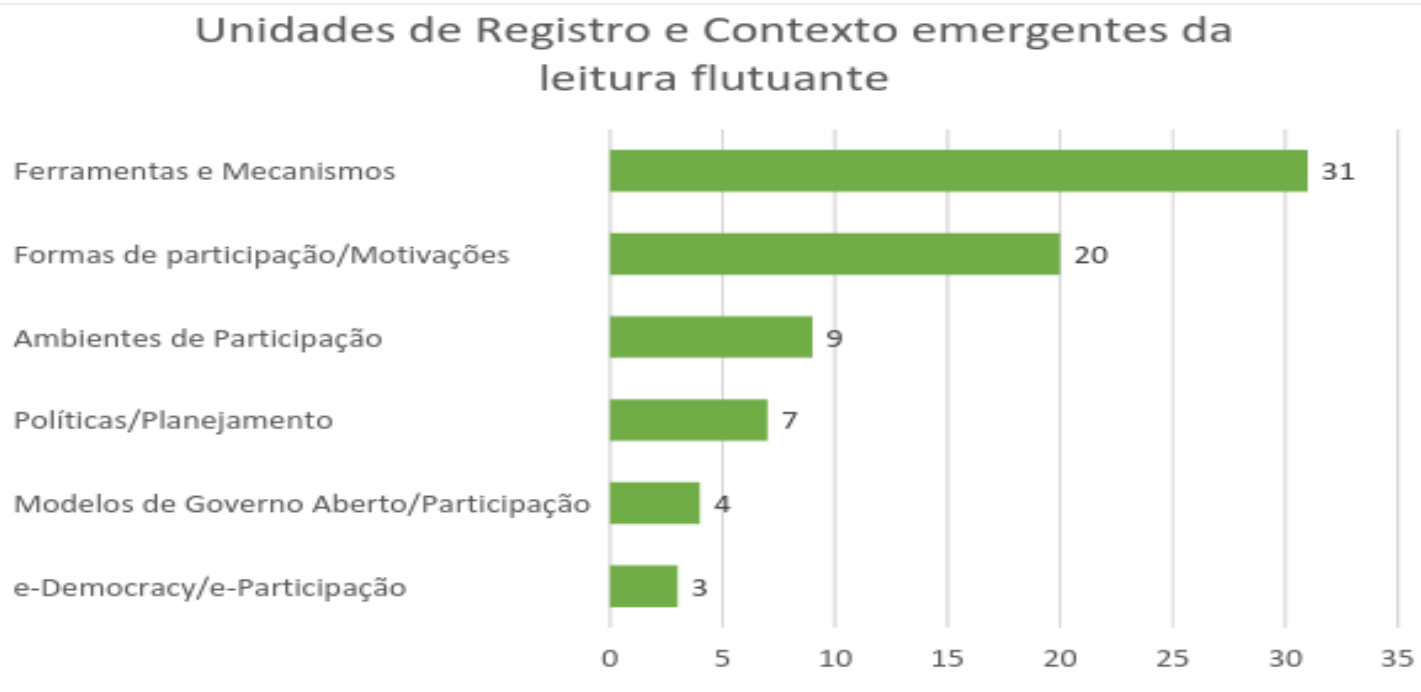

Fonte: Elaborado pelas autoras (2016).

Depreende-se do gráfico que os estudos buscam entender/explicar a participação popular em iniciativas de GA, tanto a partir do uso de ferramentas e mecanismos que possibilitem sua manifestação, como das formas de participação, suas motivações e ambientes, assim como explicitam políticas e modelos de participação.

Os resultados indicam que as iniciativas de Governo Aberto se encontram em estágio de elaboração, implementação e reformulação de políticas que garantem tanto o acesso aos dados abertos e informação governamental, quanto os espaços de participação nas tomadas de decisão

Revista Brasileira de Políticas Públicas e Internacionais, v.2, n.2, Dezembro/2017, pp. 103-118. 
SANCHEZ, Cristiane S. e MARCHIORI, Patrícia Z. A. Participação Popular no Contexto das iniciativas de Governo Aberto: revisão sistemática da literatura

governamental (Caridad Sebastián \& Martínez Cardama, 2016; Vallès Navarro et al., 2015). Neste cenário, a participação no contexto de Governo Aberto é assegurada por meio de espaços, ferramentas e tecnologias de informação e comunicação, principalmente por meio da Internet, redes sociais e aplicativos. Os estudos indicam que a Internet é um canal de comunicação entre representantes e representados (Cortes-Selva \& Perez-Escolar, 2016).

As tecnologias de comunicação e informação, o uso de ferramentas e mecanismos de participação, tais como aplicativos, sites, redes e mídias sociais, têm possibilitado a aproximação governo e governados (Baka, 2016). Este aspecto tem o potencial de impactar as próprias ferramentas, pois quanto mais o cidadão participa - via o acesso à informação - mais passa a ter a possibilidade de não somente opinar, mas também colaborar para a tomada de decisão na gestão pública (Mergel \& Desouza, 2013; Salim \& Haque, 2015; Stamati, Papadopoulos, \& Anagnostopoulos, 2015).

\subsection{Elementos que caracterizam a participação no contexto de Governo Aberto}

A partir da análise dos documentos foi possível responder à questão norteadora deste estudo, uma vez que foram identificados elementos e impactos que caracterizam a participação popular no contexto das iniciativas de Governo Aberto, conforme ilustrado na Tabela 2.

Tabela 2: Elementos emergentes da análise dos documentos sobre participação popular em iniciativas de Governo Aberto

\begin{tabular}{|c|c|c|c|}
\hline & & & Continua \\
\hline $\begin{array}{l}\text { Autores em } \\
\text { ordem } \\
\text { cronológica }\end{array}$ & Elementos & Características & Impactos \\
\hline $\begin{array}{l}\text { Prieto-Martín; } \\
\text { Ramírez-Alujas } \\
\text { (2014); } \\
\text { Bonsón; Royo; } \\
\text { Ratkai (2015); } \\
\text { Wijnhoven; } \\
\text { Ehrenhard; }\end{array}$ & $\begin{array}{l}\text { Ferramentas e } \\
\text { Mecanismos }\end{array}$ & $\begin{array}{l}\text { Internet como canal de } \\
\text { comunicação entre } \\
\text { representantes e } \\
\text { representados; } \\
\text { TIC; } \\
\text { Aplicativos; } \\
\text { Serviços por telefone; }\end{array}$ & $\begin{array}{l}\text { Acesso à informação; } \\
\text { Colaboração governo e } \\
\text { cidadãos; } \\
\text { Empoderamento do } \\
\text { cidadão; } \\
\text { Elementos } \\
\text { facilitadores da }\end{array}$ \\
\hline
\end{tabular}

Revista Brasileira de Políticas Públicas e Internacionais, v.2, n.2, Dezembro/2017, pp. 103-118. 
SANCHEZ, Cristiane S. e MARCHIORI, Patrícia Z. A. Participação Popular no Contexto das iniciativas de Governo Aberto: revisão sistemática da literatura

\begin{tabular}{|c|c|c|c|}
\hline $\begin{array}{l}\text { Kuhn (2015); } \\
\text { Baka (2016); } \\
\text { Kaigo; Okura } \\
\text { (2016). }\end{array}$ & & $\begin{array}{l}\text { Portais de dados abertos; } \\
\text { Plataformas de interação para } \\
\text { conversa online com } \\
\text { representantes } \\
\text { governamentais. }\end{array}$ & participação. \\
\hline $\begin{array}{l}\text { Caridad } \\
\text { Sebastian; } \\
\text { Martinez } \\
\text { Cardama } \\
\text { (2016). }\end{array}$ & $\begin{array}{l}\text { Formas de } \\
\text { participação }\end{array}$ & $\begin{array}{l}\text { Ativa - por meio de } \\
\text { representações e coletivos; } \\
\text { Passiva - por meio de acesso } \\
\text { à informação e ferramentas. }\end{array}$ & $\begin{array}{l}\text { Controle social; } \\
\text { Accountability } \\
\text { (Prestação de contas); } \\
\text { Maior confiança nos } \\
\text { governos; } \\
\text { Participação efetiva } \\
\text { em assuntos públicos; } \\
\text { Elaboração de } \\
\text { políticas em } \\
\text { colaboração governo- } \\
\text { cidadão. }\end{array}$ \\
\hline $\begin{array}{l}\text { Prieto-Martín, } \\
\text { Ramírez-Alujas } \\
\text { (2014); } \\
\text { Zavattaro, } \\
\text { Sementelli } \\
\text { (2014); }\end{array}$ & Agente/atores & $\begin{array}{l}\text { Cidadão; } \\
\text { Sociedade Civil Organizada; } \\
\text { Gestores. }\end{array}$ & $\begin{array}{l}\text { Sociedade } \\
\text { empoderada. }\end{array}$ \\
\hline $\begin{array}{l}\text { Porwol; Ojo; } \\
\text { Breslin (2016); } \\
\text { Jho; Song; } \\
\text { (2015). }\end{array}$ & $\begin{array}{l}\text { e-Democracy/e- } \\
\text { Participação }\end{array}$ & $\begin{array}{l}\text { Novas formas de } \\
\text { representação; } \\
\text { Novas formas de gestão. }\end{array}$ & Arranjos políticos. \\
\hline
\end{tabular}

Estes elementos, em conjunto, oferecem um panorama de como a participação é efetivada, mediada e oportunizada dentro do contexto das iniciativas de Governo Aberto comentadas no corpus analisado.

\subsection{Níveis e dimensões da participação em Governo Aberto}

Os estudos de Daniels, Lawrence \& Alig (1996); Linders (2012); Mergel (2013); Harrison \& Sayogo (2014); Prieto-Martín \& Ramírez-Alujas (2014); Zavattaro \& Sementelli (2014); Reddick, Chatfield \& Jaramillo (2015); Zavattaro, French \& Mohanty (2015); Cortés-Selva \& Pérez-Escolar (2016); Majumdar ([2016]); sendo que alguns de forma mais outros menos detalhada, apontam o

Revista Brasileira de Políticas Públicas e Internacionais, v.2, n.2, Dezembro/2017, pp. 103-118. 
SANCHEZ, Cristiane S. e MARCHIORI, Patrícia Z. A. Participação Popular no Contexto das iniciativas de Governo Aberto: revisão sistemática da literatura

estudo de (Arnstein, 1969) como precursor no sentido de representar os níveis de participação cidadã no contexto político.

O estudo de Arnstein (1969) propôs uma representação composta por oito degraus de participação (Figura 2), que concebe uma escada de participação, que simboliza os níveis de participação cívica existentes em qualquer estrutura política, social ou econômica (Arnstein, 1969). Estes degraus começam no estágio de “Não participação”, chamados de Manipulação (1) e Terapia (2), nos quais as pessoas não participam efetivamente do planejamento ou da realização de programas, mas os detentores do poder estabelecem alguns espaços - audiências, reuniões comunitárias, etc. - e se utilizam destes para mascarar um tipo de participação. Os degraus 3 (Informação) e 4 (Consulta) progridem para o nível chamado de "tokenismo" (“esforços simbólicos de participação”). Apesar de neste estágio os cidadãos poderem ouvir e ser ouvidos, eles não têm, ainda, o poder de assegurar que seus pontos de vista serão atendidos pelos detentores de poder. Quando a participação é restrita a esses níveis, não há acompanhamento e, portanto, não há garantia de se mudar o status quo. No degrau 5 - do “esforço simbólico de participação” (Pacificação) - o cidadão pode aconselhar, ainda que os detentores do poder continuem com o direito de decidir. O estágio superior "Poder do cidadão" inclui a tomada de decisão, representada pelo degrau 6 (Parceria), que lhes permite negociar com detentores de poder tradicionais a possibilidade de tomada de decisão com a “Delegação de Poder” (degrau 7) e o “Controle cidadão” (degrau 8), nos quais os cidadãos podem atingir pleno poder gerencial (Arnstein, 1969).

Revista Brasileira de Políticas Públicas e Internacionais, v.2, n.2, Dezembro/2017, pp. 103-118. 
SANCHEZ, Cristiane S. e MARCHIORI, Patrícia Z. A. Participação Popular no Contexto das iniciativas de Governo Aberto: revisão sistemática da literatura

Figura 2. Escada da participação cidadã.

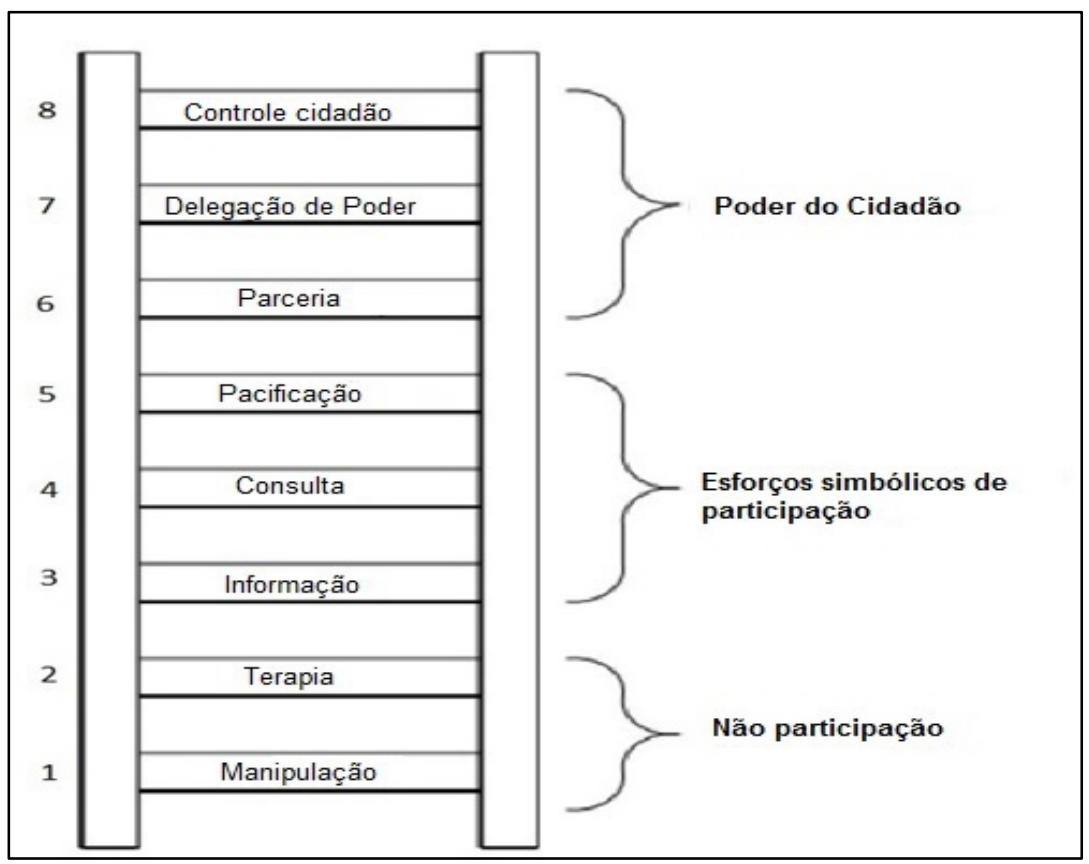

Fonte: Traduzido de Arnstein, 1969 pelas autoras (2017).

Nesta representação é possível verificar as condições que permitem ao cidadão abandonar a passividade e conquistar uma participação ativa na tomada de decisão e, efetivamente, colaborar com a gestão governamental.

A partir do estudo de Arnstein (1969), (Prieto-Martin \& Ramirez-Alujas, 2014) identificaram dimensões que possibilitam a caracterização da participação em GA, sendo estas apontadas como: dimensão “que”, que se refere à intensidade da participação; dimensão “quando”, que trata dos momentos e fases da participação; dimensão “onde”, que se refere ao nível de institucionalização da participação; dimensão “como”, que trata da participação por meio da transparência e intensidade deliberativa; e, dimensão “quem”, que se refere aos atores da participação que impactam e são afetados por esta. No modelo de Prieto-Martín \& Ramírez-Alujas (2014) se destaca uma fase anterior: a de não participação, que é denominada de “Conflito”, na qual se inclui a participação autônoma por parte daqueles interessados em uma decisão ou determinado problema. A fase de “Conflito”, difere do primeiro estágio apontado por Arnstein (“Manipulação”)

Revista Brasileira de Políticas Públicas e Internacionais, v.2, n.2, Dezembro/2017, pp. 103-118. 
SANCHEZ, Cristiane S. e MARCHIORI, Patrícia Z. A. Participação Popular no Contexto das iniciativas de Governo Aberto: revisão sistemática da literatura

pois neste apenas os tomadores de decisão podem ser mobilizados. Outra diferença está no nível, denominado por Arnstein como esforços simbólicos de participação (tokenismo), que é chamado pelos autores de Participação Consultiva, no qual a Assessoria assemelha-se ao degrau “Pacificação”, quando o cidadão se assume partícipe na tomada de decisão e passa a buscar a parceria com o governo. Ainda, no modelo de Prieto-Martín \& Ramírez-Alujas (2014) o chamado Poder do Cidadão daria espaço à Participação Colaborativa. Em suma, os ajustes elaborados pelos autores ampliam os níveis de Participação Cidadã, expandindo os “degraus” (Arnstein, 1969) que, potencialmente possibilitam a colaboração governo-cidadãos e podem tornar estes últimos agentes mais ativos no contexto das iniciativas de Governo Aberto.

\subsection{Condicionantes da participação em Governo Aberto: motivações, barreiras e desafios}

Quanto às motivações para a participação popular, estas ocorrem por meio de variados estímulos e em ambientes diferenciados, ou seja, os espaços de participação encontram-se de forma institucionalizada, via entidades coletivas que representam a sociedade civil organizada. As motivações vão desde afinidade do cidadão com o tema tratado, passando pela possibilidade de contribuição, até a influência da própria cultura em que o mesmo está inserido. Assuntos que afetam diretamente os cidadãos, tais como questões relacionadas à coleta de lixo, serviços de segurança, informações sobre creches, trânsito, têm mais apelo para que o cidadão participe ativamente, tanto na busca por dados e informações, quanto na tomada de decisão (Baka, 2016; Vallès Navarro et al., 2015; (Amichai-Hamburger et al., 2016); (Zavattaro, French, \& Mohanty, 2015))

A participação popular em contexto de Governo Aberto encontra algumas barreiras e desafios para sua efetivação. Neste sentido, a análise dos textos revelou que as Tecnologias de Informação e Comunicação e as Mídias Sociais, ao mesmo tempo em que aproximam a população dos Governos (e são ferramentas que possibilitam a colaboração entre as partes), também podem ser entraves para a participação, conforme ilustrado na Tabela 3.

Tabela 3: Condicionantes (desafios e barreiras) para a participação em iniciativas de Governo Aberto

Revista Brasileira de Políticas Públicas e Internacionais, v.2, n.2, Dezembro/2017, pp. 103-118. 
SANCHEZ, Cristiane S. e MARCHIORI, Patrícia Z. A. Participação Popular no Contexto das iniciativas de Governo Aberto: revisão sistemática da literatura

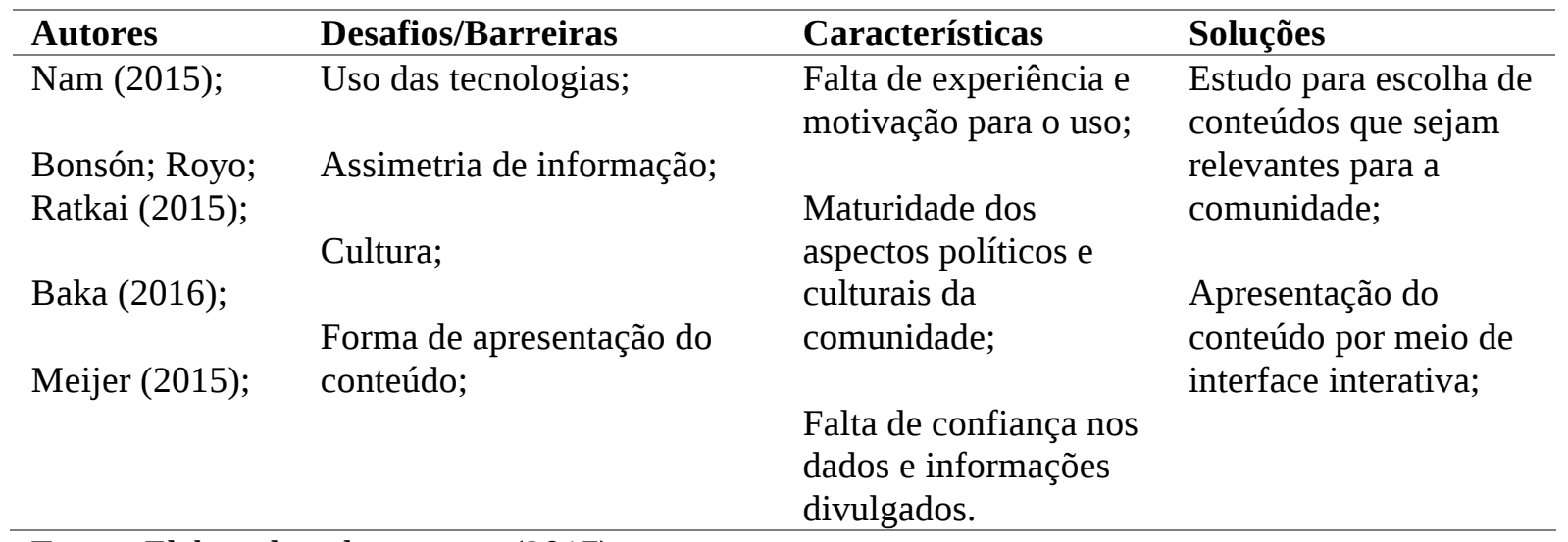

Fonte: Elaborado pelas autoras (2017).

Essas condicionantes verificadas nos estudos que compuseram o corpus de análise tendem a impactar na participação na gestão governamental - principalmente na elaboração, implementação e/ou reformulação de Políticas, bem como na participação direta no Planejamento governamental.

\section{Considerações Finais}

A sistematização dos elementos que caracterizam a participação em contexto das iniciativas de GA possibilitou o entendimento de que a participação se encontra em estágio de implementação e reformulação de políticas. Tais políticas, quando implantadas/reformuladas podem garantir o acesso aos dados abertos e informação governamental, bem como assegurar a participação efetiva da população na elaboração de políticas de Governo Aberto.

A compreensão destes elementos pode contribuir para a estruturação mais eficiente da divulgação de dados governamentais, voltadas aos interesses dos cidadãos; na formulação de aplicativos, portais e mídias para ampliação do cidadão na gestão governamental; na observação das motivações que conduzem os cidadãos a participarem; assim como no fortalecimento da colaboração governo-cidadão, cidadão-governo, governo-negócios e negócios cidadãos, de modo a contribuir para os avanços políticos, sociais e econômicos.

Para tanto deve-se considerar as diversas correntes que buscam conceituar o termo participação, assim como a contemporaneidade das propostas de GA. Esta sistematização, portanto, pode auxiliar os interessados em promover a participação popular no contexto das iniciativas de Revista Brasileira de Políticas Públicas e Internacionais, v.2, n.2, Dezembro/2017, pp. 103-118. 
SANCHEZ, Cristiane S. e MARCHIORI, Patrícia Z. A. Participação Popular no Contexto das iniciativas de Governo Aberto: revisão sistemática da literatura

GA, de modo que os pilares e premissas, tais como transparência, controle social, colaboração, possam ser alcançados.

Estudos empíricos podem auxiliar a construir o quadro geral de participação. Porém, caso se apresentem realidades pontuais, estas podem não denotar o arcabouço necessário para construção do conceito, especialmente no contexto de iniciativas de GA. Neste sentido, recomenda-se que em estudos futuros sejam adotados os marcos regulatórios de cada país, uma vez que as iniciativas se encontram em estágios diferenciados de implantação ou avaliação.

A continuidade de estudos nesta temática pode, a médio prazo, contribuir para a criação um quadro conceitual que embase discussões, tanto em nível prático como teórico, relativas a imbricação das políticas públicas com os dados abertos e a participação cidadã.

\section{Referências}

Amichai-Hamburger, Y., Gazit, T., Bar-Ilan, J., Perez, O., Aharony, N., Bronstein, J., \& Sarah Dyne, T. (2016). Psychological factors behind the lack of participation in online discussions. Computers in Human Behavior, 5art A, 268-277. doi:http://dx.doi.org/10.1016/j.chb.2015.09.009

Arnstein, R. S. (1969). A Ladder of Citizen Participation. JAIP, Vol. 35, pp. 216-224.

Attard, J., Orlandi, F., Scerri, S., \& Auer, S. (2015). A systematic review of open government data initiatives. Government Information Quarterly, 399-418. doi:http://dx.doi.org/10.1016/j.giq.2015.07.006

Baka, V. (2016). Co-creating an open platform at the local governance level: How openness is enacted in Zambia. Government Information Quarterly. doi:http://dx.doi.org/10.1016/j.giq.2016.10.001

Bardin, L. (2016). Análise de conteúdo (revista e ampliada ed.). São Paulo, SP: Edições 70.

Caridad Sebastián, M., \& Martínez Cardama, S. (2016). Gobierno y Parlamento abierto: la participación ciudadana en el tratamiento y visualización de la información pública. Government and Open Parliament: Citizens Participation in the Treatment and Visualization of the Public Information., 39(1), 47-56. doi:10.17533/udea.rib.v39n1a06

Cortes-Selva, L., \& Perez-Escolar, M. (2016). Civic participation and interactive documentaries: a contribution to the open government model. Profesional De La Informacion, 25(4), 578-587. doi:10.3145/epi.2016.jul.07

Foti, J. ([2014]). Independent Reporting Mechanism Technical Paper 1. In: Open Government Partnership.

Revista Brasileira de Políticas Públicas e Internacionais, v.2, n.2, Dezembro/2017, pp. 103-118. 
SANCHEZ, Cristiane S. e MARCHIORI, Patrícia Z. A. Participação Popular no Contexto das iniciativas de Governo Aberto: revisão sistemática da literatura

Heredia, B. M. A. D., Bezerra, M. O., \& Palmeira, m. (2012). Introdução. In Política, governo e participação popular : conselhos, orçamento participativo e outras experiências. Rio de Janeiro, RJ: 7 Letras.

Mergel, I., \& Desouza, K. C. (2013). Implementing Open Innovation in the Public Sector: The Case of Challenge.gov. Public Administration Review, 73(6), 882-890. doi:10.1111/puar.12141

Open Government Declaration, (2011). Retrieved from https://www.opengovpartnership.org/opengovernment-declaration

Prieto-Martin, P., \& Ramirez-Alujas, A. (2014). Characterizing Citizen Participation within Open Government. Revista Del Clad Reforma Y Democracia(58), 61-+.

Rowe, G., \& Frewer, L. J. (2005). A Typology of Public Engagement Mechanisms. Science, Technology, \& Human Values, 30(2), 251-290. doi:10.1177/0162243904271724

Salim, F., \& Haque, U. (2015). Urban computing in the wild: A survey on large scale participation and citizen engagement with ubiquitous computing, cyber physical systems, and Internet of Things. International Journal of Human-Computer Studies, 81, 31-48. doi:http://dx.doi.org/10.1016/j.ijhcs.2015.03.003

Silva, C. M. C. (2015). Uso de mídia social governamental para promover engajamento entre cidadãos e governo federal. (Mestrado), Universidade de São Paulo, São Paulo, SP. Retrieved from http://www.teses.usp.br/teses/disponiveis/100/100131/tde-01052015-103725/pt-br.php

Stamati, T., Papadopoulos, T., \& Anagnostopoulos, D. (2015). Social media for openness and accountability in the public sector: Cases in the Greek context. Government Information Quarterly, 32(1), 12-29. doi:10.1016/j.giq.2014.11.004

The Cochrane Database of Systematic Reviews. (2017). Retrieved from http://www.cochranelibrary.com/cochrane-database-of-systematic-reviews/index.html

Vallès Navarro, R., Costa Vilar, N., Davins Miralles, J., Garcia Cirera, M., Hernando Ortega, M. R., \& Iniesta Blasco, C. (2015). El Consejo Consultivo de Pacientes de Cataluña: la voz directa del paciente en las políticas de salud en Cataluña. Medicina Clínica, 145, Supplement 1, 27-30. doi:http://dx.doi.org/10.1016/S0025-7753(15)30034-8

Yu, H., \& Robinson, D. G. (2012). The New Ambiguity of “Open Government” - UCLA Law Review. UCLA Law Review, 59, 178-208.

Zavattaro, S. M., French, P. E., \& Mohanty, S. D. (2015). A sentiment analysis of U.S. local government tweets: The connection between tone and citizen involvement. Government Information Quarterly, 32(3), 333-341. doi:http://dx.doi.org/10.1016/j.giq.2015.03.003

Revista Brasileira de Políticas Públicas e Internacionais, v.2, n.2, Dezembro/2017, pp. 103-118. 